\title{
AUXILIAR DE DOCENTE NO CONTEXTO DO ENSINO TECNOLÓGICO: FINALIDADES E ATRIBUIÇÕES
}

https://dx.doi.org/10.5902/2318133867834

\author{
Julio Francisco Rodrigues de Sousa ${ }^{1}$ \\ Marília Macorin de Azevedo \\ Paulo Roberto Prado Constantino ${ }^{3}$
}

\begin{abstract}
Resumo
É crescente na literatura a importância da educação profissional e tecnológica para o desenvolvimento mundial e, nesse sentido, faz-se necessário que exista uma estrutura com profissionais preparados para o atendimento de seus objetivos e propostas. Nesse contexto, 0 presente trabalho tem por objetivo apresentar, na percepção de docentes e auxiliares de docente, uma avaliação das atribuições destes em duas instituições de educação tecnológica pública nos municípios de São Paulo e Sorocaba. Realizou-se uma pesquisa de objetivo descritivo, abordagem qualitativa, natureza aplicada e com procedimento de pesquisa de campo na qual se aplicou um questionário eletrônico estruturado a todos os docentes e auxiliares de docente dessas unidades, em que foram relacionadas as atribuições desses profissionais, e os respondentes apontaram, segundo escala de Likert, quais são suas percepções acerca do tempo investido e qual a importância da mesma no cotidiano da estrutura de ensino. Os dados indicam que a maioria dos profissionais possuem uma percepção positiva acerca da atuação do auxiliar de docente, considerando a maior parte das atribuições com importância muito alta para o sucesso da educação profissional e tecnológica. As atribuições dos grupos de atividades técnicooperacionais e de relacionamento com os alunos foram as mais relevantes, sendo que as outras foram entendidas como complementares e menos importantes nesse contexto.
\end{abstract}

Palavras-chave: educação profissional e tecnológica; auxiliar de docente; avaliação; gestão escolar.

\section{TEACHING ASSISTANT IN THE CONTEXT OF TECHNOLOGICAL EDUCATION: PURPOSES AND ATTRIBUTIONS}

\section{Abstract}

There is a growing importance in the literature related to professional and technological education in the world development and, in this sense, it must have a structure with professionals prepared to meet their objectives and proposals. In this context, the present work aims to present, in the perception of teachers and teaching assistants, an assessment of their attributions in two public technological education institutions in São Paulo and Sorocaba municipalities. Research with a descriptive objective, qualitative approach, applied nature and a field research procedure was carried out, in which a structured electronic questionnaire was applied to all teachers and teaching assistants of these units, the attributions of these professionals were related and the respondents indicated, according to the Likert scale, their perceptions about the time invested and its

\footnotetext{
${ }^{1}$ Centro Paula Souza, Brasil. E-mail: link julio@hotmail.com.

2 Centro Paula Souza, Brasil. E-mail: marilia.azevedo@fatec.sp.gov.br.

${ }^{3}$ Centro Paula Souza, Brasil. E-mail: paulo.constantino@cps.sp.gov.br.
} 
importance in the daily life of the teaching structure. Data indicated that most professionals have a positive perception about the role of teaching assistants, considering that most of the assignments are very important for the success of professional and technological education. The attributions of the groups of technical-operational activities and relationship with students were the most relevant, while the others were understood as complementary and less important in this context.

Key-words: professional and technological education; teaching assistant; evaluation; school management.

\section{Introdução}

1 educação profissional e tecnológica é uma modalidade de ensino relevante em nível nacional e internacional. É reconhecida pela literatura como voltada ao ensino profissionalizante de qualidade aos educandos com vistas ao atendimento das demandas tão dinâmicas e complexas da industrialização (Quevedo, 2011; Oliveira; Cóssio, 2013; Feitoza; Duduchi, 2016; Vieira; Souza Júnior, 2016; Magalhães; Castioni, 2018). Porém, segundo Uemura (2016), para que sejam atingidos os objetivos preconizados a essa modalidade de educação, deve-se contar com uma estrutura adequada e engajada que atenda aos requisitos necessários para esse fim, e é nesse contexto que se encontra o auxiliar de docente.

Esse profissional se mostra importante na estrutura brasileira de EPT pelas suas atribuições no âmbito da organização de atividades e manutenção de estruturas físicas em geral, atuando como facilitador do processo de ensino e aprendizagem e estando próximo das práticas docentes do setor. Pensando no caso específico das faculdades de tecnologia e escolas técnicas do Centro Estadual de Educação Tecnológica Paula Souza (Ceeteps, 2013), o auxiliar de docente atua dando suporte às atividades técnicas e práticas de variadas disciplinas e estabelecendo relações mais próximas aos discentes, assessorando na aprendizagem efetiva dos mesmos. Verifica-se que a carreira nessas instituições está voltada ao preenchimento de lacunas de acompanhamento e efetividade da aprendizagem tecnológica pelos alunos, sendo nesse contexto um profissional com conhecimento e bagagem científico-tecnológica apropriada para orientar os alunos e assessorar os docentes.

Considerando o exposto, o presente trabalho apresenta uma avaliação das atribuições e finalidades dos auxiliares de docente em duas instituições de educação pública superior nos municípios de São Paulo e Sorocaba, segundo a percepção de docentes e auxiliares de docente.

\section{Educação profissional e tecnológica: discussões e estruturas}

A educação profissional e tecnológica, conforme a definição do Centro Europeu para o Desenvolvimento da Formação Profissional - Cedefop -, é a modalidade de ensino que tem como objetivo dotar pessoas de conhecimentos teóricos e práticos, capacidades ou competências exigidas por profissões específicas ou pelo mercado de trabalho (Magalhães; Castioni, 2018). Quevedo (2011) relembra também a Lei de Diretrizes e Bases da Educação (Brasil, 1996), que define a EPT como 
domínio operacional de determinado fazer, acompanhado da compreensão global do processo produtivo, com apreensão do saber tecnológico, valorização da cultura do trabalho e mobilização dos valores necessários à tomada de decisões. (Quevedo, 2011, p. 154)

O desenvolvimento dessa modalidade acompanha relações muito antigas entre trabalho e educação e, segundo Quevedo (2011), a partir da Revolução Industrial, a escola se tornou um agente direto da incorporação das funções intelectuais no processo produtivo e consequente universalização do acesso ao conhecimento. Porém, mesmo no século 20, a educação profissional ainda era considerada como de segunda categoria, apontada por Feitoza e Duduchi (2016) como "assistencialista, voltada aos menos favorecidos, propondo-se a ensinar a trabalhar e inserir no mercado a classe dos 'desvalidos'” (p. 495). Isso só mudou mais efetivamente na década de 1980, com as novas exigências por capacidades em inovação, criatividade e gestão dos trabalhadores, alçando a educação profissional e tecnológica a um novo nível.

No Brasil, a EPT é regulamentada pela Constituição Federal de 1988 (Brasil, 2021), com as atualizações da Emenda Constitucional n. 85/2015, pela LDB (Brasil, 1996), regulamentada pelo Decreto n. 5.154/2004 e alterada pela Lei n. 11.741/2008 (Brasil, 2008). Pela lei n. 13.415/2017 (Brasil, 2017) se instituiu novas alterações aos dispositivos relacionados à modalidade no Brasil. Cabe destacar que a Secretaria de Educação Profissional e Tecnológica - Setec/MEC - é a responsável por formular, planejar, coordenar, implementar, monitorar e avaliar políticas públicas de educação profissional e tecnológica no Brasil (MEC, 2020).

Na perspectiva do ensino superior tecnológico, segundo dados do Inep (2020), no Censo da Educação Superior 2019, a quantidade de ingressos nessa modalidade representou $23 \%$ do total de ingressos do país em ensino superior, portanto, quase um quarto desse total. Os dados chamam atenção também para o crescimento do número de cursos de educação à distância: em 2009, 28,5\% das matrículas do país em cursos de EPT eram em EAD, enquanto $71,5 \%$ eram em cursos presenciais. Já em $2019,58,1 \%$ das matrículas foram em EAD, enquanto os outros $41,9 \%$ foram em cursos presenciais (Inep, 2020). Nas redes públicas, o Censo evidenciou que a maioria das matrículas ocorre em cursos presenciais, enquanto que a iniciativa privada foi a maior responsável pela difusão da modalidade EAD.

A literatura destaca um crescimento das estruturas relacionadas a EPT no país nos últimos anos. Segundo Magalhães e Castioni (2018), nos anos de 2000 e 2007, o governo criou os chamados institutos federais para alavancar a EPT como uma estratégia para o país através do Plano de Desenvolvimento da Educação em cidades com mais de 50 mil habitantes. Porém, muito embora Oliveira e Cóssio (2013) defendam que essa expansão foi fundamental para o país, universalizando o acesso à educação, Magalhães e Castioni (2018) discutem que os interesses relacionados a essa expansão ainda são difusos e indefinidos, havendo ainda pouco entendimento sobre seu papel e escassez de estudos que indiquem a efetividade dos investimentos realizados, culminando em espaços com propostas diferentes dos conceitos vocacionais praticados em outros países, como Alemanha e Suíça. 
Além dos IF e outros órgãos de nível federal como os centros federais de educação tecnológica e as escolas técnicas federais, existem redes estaduais proeminentes no segmento, como o Centro Estadual de Educação Tecnológica Paula Souza - Ceeteps -, mantenedor de escolas técnicas e faculdades de tecnologia que promove 0 desenvolvimento gratuito da EPT. No caso das Fatecs, foco do presente estudo, a excelência também é reconhecida pela literatura e é resultante de um amplo desenvolvimento histórico. Peterossi (2005) ressalta que a ação institucional das Fatecs acompanhou as políticas públicas de desenvolvimento socioeconômico do estado de São Paulo e que elas desempenham um papel estratégico na inovação e melhoria da qualidade dos processos produtivos das empresas ao formar profissionais de alta qualificação e competência técnica. Por fim, deve-se lembrar a existência de outras redes atuantes no segmento, como o Serviço Nacional de Aprendizagem Industrial, que sustentam e auxiliam no crescimento dos índices de difusão da EPT.

\section{O auxiliar de docente no contexto da educação profissional e tecnológica}

A literatura reserva atenção à atuação docente na educação profissional e tecnológica, porém essa atenção é menor no caso dos auxiliares de docente, mesmo compreendendo a relevância destes na estrutura de EPT. No caso deste trabalho, serão consideradas especialmente as normas relacionadas à atuação do auxiliar de docente no Centro Estadual de Educação Tecnológica Paula Souza - Ceeteps. Esse profissional, segundo a lei complementar n. 1.044, de 13 de maio de 2008 (São Paulo, 2008), alterada pelas leis complementares n. 12.40/2014 e n. 1.343/2019 (São Paulo, 2014; 2019), integra o quadro de empregados públicos do Ceeteps com carga horária regulamentada e definida nos níveis de EPT referidos. Sua representação sindical é feita principalmente pelo Sindicato de Trabalhadores do Centro Paula Souza - Sinteps - e seu Código Brasileiro de Ocupação, que consta apenas em tabela exclusiva do Centro Paula Souza, é 2394-10.

A Deliberação Ceeteps n. 08/2014 (Ceeteps, 2014), estipula o rol de atribuições do auxiliar de docente nos níveis técnico médio e tecnológico superior da autarquia. É possível perceber, por meio dessa deliberação, a quantidade de papeis desempenhados e quão vasta pode ser a atuação do profissional na cadeia de EPT do Centro Paula Souza. Essa variabilidade, por sua vez, não é pontual, mas acompanha a evolução do cargo com o tempo. Anteriormente, conforme o regimento unificado da instituição, no decreto n. 17.027/1981 (São Paulo, 1981), discriminavam-se categorias de docentes professor pleno, professor associado, professor assistente e professor auxiliar - e, além deles, mencionavam-se os 'auxiliares de magistério', quais sejam os cargos de Instrutor e de Auxiliar de docente.

Outros mecanismos legais da época definiram critérios remuneratórios e correlações salariais entre as categorias, o que implicava em um plano de carreira do auxiliar até o docente. Porém, em momentos posteriores, ocorreu uma convergência de atribuições em um único cargo, denominado unicamente como 'Auxiliar de docente', mas que desempenha funções cumulativas do Instrutor e do Auxiliar de docente da antiga carreira. Também novos critérios remuneratórios foram estipulados pelas leis complementares $\mathrm{n}$. 1.044/2008 (São Paulo, 2008) e n. 1.148/2011 (São Paulo, 2011), passando a não existir mais a correlação direta e proporcional entre os docentes e auxiliares de docente e 
inexistindo também um plano de carreira entre os mesmos. Dessa forma, a convergência ora constatada resultou em confluência de atribuições antes diferentes em torno de um único cargo.

O conceito, atuação, competências e pré-requisitos do auxiliar de docente divergem conforme os locais do mundo, sendo muitas vezes diferentes daqueles existentes na estrutura brasileira de EPT. Na literatura, a figura mais próxima do auxiliar de docente em questão no presente trabalho é, segundo Malara (2008), a dos teaching assistants, muito próximos a professores auxiliares em instituições dos Estados Unidos, doravante 'professores auxiliares de classe'. Esses profissionais, conforme citado por Malara (2008), são uma categoria muito comum na EPT de diversos níveis nos EUA (Reeves et al, 2016; Justice; Zieffler; Garfield, 2017; Alexander; Smith, 2018; Marshman Et Al, 2018; Rutledge et al, 2018), mas não se restringem a esse país, havendo casos em Israel (Alhija; Fresko, 2018), Taiwan (Chou; Huang; Lin, 2011; Liao, 2018), Reino Unido (Wren, 2017) e outros não evidenciados neste trabalho.

Uma perspectiva dos teaching assistants é a de estudantes em certo nível do curso que os permite realizar atividades de assessoria aos docentes, chegando a ministrar aulas e participar de cargas horárias específicas de aulas como ouvintes. Eles facilitam a condução e execução das aulas e auxiliam diretamente nas demandas dos estudantes, o que estabelece uma conexão de atribuições entre esses assistentes e os auxiliares de docente em questão no presente estudo. Porém, ressalta-se a diferença que, no Brasil, exige-se formação e experiência dos auxiliares de docente, enquanto que, em outras estruturas educacionais, esses profissionais estão em formação.

Alguns estudos avaliaram percepções a respeito da atuação desses profissionais segundo diferentes enfoques. Alhija e Fresko (2018) e Liao (2018) avaliaram as percepções de estudantes quanto à atuação dos assistentes e, a partir dos questionários aplicados, destacaram altos índices de satisfação atribuídos pela comunidade discente, o que Alhija e Fresko (2018) afirmam ser ainda mais expressivo em cursos da área de Ciências Exatas. Benefícios como bons esclarecimentos e boa didática de ensino são citados nessas pesquisas, e um ponto adicional colocado em questão por Alhija e Fresko (2018) é que as atuações variam conforme a área do conhecimento, em qualquer local que se avalie, uma vez que existem requisitos técnicos e conhecimentos específicos necessários a esses profissionais para a entrega de maior qualidade em seu trabalho.

Segundo Alexander e Smith (2018), é necessário atribuir aos auxiliares de docente um treinamento pedagógico e experiência de aulas, de forma que se garanta a qualidade da sua atuação nos modelos pedagógicos de diferentes níveis. Nesse sentido, nos modelos internacionais de atuação dos teaching assistants, a literatura elucida o caráter de aprendizes com competência para orientar em certos momentos, porém, no Brasil, a triagem geralmente realizada, que exige formação e experiência na modalidade requerida, tende a amenizar esse ponto, uma vez que as competências técnicas necessárias já são exigidas de entrada e, assim, pode contar como um ponto de vantagem do modelo brasileiro frente a outros países.

Portanto, de maneira geral, em comparação a práticas internacionais, ressalvadas as diferenças de contextos e graus de experiência - os teaching assistants como estudantes em treinamento e os auxiliares de docente com formação e experiência para suporte ao docente -, os objetivos da atuação desses profissionais são semelhantes,

\begin{tabular}{|l|l|l|l|l|l|}
\hline Regae: Rev. Gest. Aval. Educ. & Santa Maria & v. 11 & n. 20 & e67834, p. 1-16 & 2022 \\
\hline
\end{tabular}


estando alinhados a expectativas de facilitação do ensino e aprendizagem dos estudantes em diferentes níveis, bem como de assessoria às atividades gerais dos docentes. Assim, deseja-se reafirmar nesse trabalho a relevância desse profissional na estrutura da EPT, o que o justifica como foco do presente trabalho.

\section{Método}

A presente pesquisa é de objetivo descritivo, abordagem qualitativa, natureza aplicada e com procedimento de pesquisa de campo na qual se aplicou um questionário eletrônico estruturado a todos os docentes e auxiliares de docente das Faculdades de Tecnologia de São Paulo e de Sorocaba, sendo que as mesmas foram selecionadas por terem um contingente significativo desses profissionais em comparação às demais unidades de ensino superior da autarquia em análise. O questionário relacionou as atribuições do auxiliar de docente segundo a deliberação Ceeteps n. 8, de 10 de julho de 2014:

A) Instruir alunos na execução das práticas operacionais específicas de tarefas nos laboratórios e oficinas, orientando-os nas técnicas de utilização de máquinas, ferramentas, instrumentos, aparelhos, etc., para habilitá-los à análise do desempenho na execução da tarefa; B) Efetuar demonstração das técnicas operacionais, manipulando ferramentas, máquinas, instrumentos e equipamentos; C) Fornecer dados e informações necessárias ao trabalho de cada aluno, para possibilitar o desenvolvimento das operações dentro das especificações exigidas; D) Interpretar e explicar, individualmente ou em grupo, detalhes de desenho ou das especificações escritas para orientação do aluno sobre o roteiro e a forma correta da execução do trabalho; E) Diligenciar no sentido de que os alunos se utilizem adequadamente das máquinas, ferramentas, instrumentos, equipamentos, etc; F) Providenciar a preparação do local de trabalho, dos materiais, ferramentas, instrumentos, máquinas e equipamentos a serem utilizados, verificando as condições dos mesmos, o estado de conservação de todos os equipamentos e cuidados de segurança dos alunos, para assegurar a execução correta das tarefas e operações programadas; G) Observar e fazer observar, permanentemente, as normas de higiene e segurança do trabalho em todos os locais; $\mathrm{H}$ ) Comunicar ao superior hierárquico as irregularidades e os problemas constatados, de qualquer ordem; I) Colaborar para o bom funcionamento dos laboratórios e das oficinas; J) Cuidar da preparação dos materiais de consumo, nos laboratórios, quando originários do almoxarifado; K) Providenciar e/ou confeccionar corpos de prova para ensaios de materiais de uso nos laboratórios e nas oficinas; L) Participar de reuniões sempre que convocado; M) Manter-se atualizado com o desenvolvimento técnico, científico ou cultural, relativo ao seu campo de atividade; N) Frequentar os treinamentos e cursos de atualização, extensão e outros promovidos pela unidade de ensino; O) Cuidar da instalação, manutenção e reparação de máquinas, equipamentos e instalações de laboratórios; P) Cuidar da organização do setor de manutenção e almoxarifado pertencentes aos laboratórios e suas instalações; Q) Colaborar nos trabalhos gerais de instalação, manutenção e reparação, realizados na unidade de ensino; R) Zelar pela manutenção e conservação das máquinas, ferramentas, instalações e equipamentos de trabalho; S) Colaborar com o docente em programas de extensão universitária à comunidade. (Ceeteps, 2014). 
Com base nessa relação, cada respondente teve a oportunidade de assinalar, conforme escalas Likert de cinco pontos, qual o tempo gasto pelo auxiliar de docente para desempenhar as atribuições em seu cotidiano e qual a importância [segundo sua percepção] de cada atribuição dentro da atuação dos profissionais. Aplicou-se a pesquisa utilizando um Formulário Google enviado via e-mail aos profissionais das unidades consideradas. A Fatec/SP possuía, até dezembro de 2020, 264 docentes e 67 auxiliares de docente, enquanto a Fatec Sorocaba possuía, no mesmo período, 108 docentes e 32 auxiliares de docente, especialistas de diferentes áreas do conhecimento.

Posteriormente, dispondo-se dos dados coletados, realizou-se uma análise estatística descritiva para identificar pontos de disparidade e concordância entre os respondentes em termos de cargos: percepção dos docentes versus percepção dos auxiliares de docente. Além das análises das percepções dos docentes e auxiliares de docente, preliminarmente foram realizadas entrevistas semiestruturadas com os diretores das faculdades de tecnologia de São Paulo e de Sorocaba, e algumas informações oriundas dessas entrevistas foram eventualmente utilizadas nas discussões.

\section{Resultados e discussões}

Registrou-se um total de 85 respostas de profissionais das faculdades de tecnologia de São Paulo e Sorocaba. Num primeiro momento, na tabela 1 se apresenta os percentuais relativos aos tempos de experiência e faixas etárias dos respondentes, e na tabela 2 se apresenta o percentual relativo de respondentes de cada unidade em função do total de profissionais daquele cargo na mesma.

Tabela 1 -

Tempo de experiência e faixas etárias dos respondentes por faixas

\begin{tabular}{c|c|c|c|c|c}
\hline \multicolumn{6}{c}{ Tempo de experiência } \\
\hline Docentes & FATEC/SP & $\begin{array}{c}\text { FATEC } \\
\text { Sorocaba }\end{array}$ & $\begin{array}{c}\text { Auxiliares de } \\
\text { docente }\end{array}$ & FATEC/SP & $\begin{array}{c}\text { FATEC } \\
\text { Sorocaba }\end{array}$ \\
\hline Total & 23 & 29 & Total & 17 & 16 \\
\hline Até 2 anos & $1(4,35 \%)$ & $0(0,00 \%)$ & Até 2 anos & $3(17,65 \%)$ & $2(12,5 \%)$ \\
\hline De 3 a 5 anos & $0(0,00 \%)$ & $2(6,90 \%)$ & De 3 a 5 anos & $0(0,00 \%)$ & $1(6,25 \%)$ \\
\hline De 6 a 8 anos & $0(0,00 \%)$ & $1(3,45 \%)$ & De 6 a 8 anos & $9(52,94 \%)$ & $4(25,0 \%)$ \\
\hline De 9 a 11 anos & $4(17,39 \%)$ & $6(20,69 \%)$ & De 9 a 11 anos & $3(17,65 \%)$ & $7(43,75 \%)$ \\
\hline De 12 a 15 anos & $1(4,35 \%)$ & $4(13,79 \%)$ & De 12 a 15 anos & $0(0,00 \%)$ & $1(6,25 \%)$ \\
\hline 16 anos ou mais & $17(73,91 \%)$ & $16(55,17 \%)$ & 16 anos ou mais & $2(11,76 \%)$ & $1(6,25 \%)$ \\
\hline
\end{tabular}

Faixas etárias

\begin{tabular}{c|c|c|c|c|c}
\hline Docentes & FATEC/SP & $\begin{array}{c}\text { FATEC } \\
\text { Sorocaba }\end{array}$ & $\begin{array}{c}\text { Auxiliares de } \\
\text { docente }\end{array}$ & FATEC/SP & $\begin{array}{c}\text { FATEC } \\
\text { Sorocaba }\end{array}$ \\
\hline Total & 23 & 29 & Total & 17 & 16 \\
\hline 21 a 30 anos & $0(0,00 \%)$ & $2(6,90 \%)$ & 21 a 30 anos & $6(35,29 \%)$ & $6(37,5 \%)$ \\
\hline 31 a 40 anos & $0(0,00 \%)$ & $0(0,00 \%)$ & 31 a 40 anos & $5(29,41 \%)$ & $6(37,5 \%)$ \\
\hline
\end{tabular}




\begin{tabular}{c|c|c|c|c|c}
\hline 41 a 50 anos & $5(21,74 \%)$ & $2(6,90 \%)$ & 41 a 50 anos & $4(23,53 \%)$ & $2(12,5 \%)$ \\
\hline 51 a 60 anos & $7(30,43 \%)$ & $11(37,93 \%)$ & 51 a 60 anos & $1(5,88 \%)$ & $2(12,5 \%)$ \\
\hline 61 anos ou mais & $11(47,83 \%)$ & $14(48,28 \%)$ & 61 anos ou mais & $1(5,88 \%)$ & $0(0,00 \%)$ \\
\hline
\end{tabular}

Fonte: autores.

Tabela 2 -

Quantidades de respostas obtidas por unidade e por classe profissional

\begin{tabular}{c|c|c}
\hline Unidade $x$ profissional & FATEC/SP & FATEC Sorocaba \\
\hline Docentes & 23 de $264(8,71 \%)$ & 29 de $108(26,85 \%)$ \\
\hline Auxiliares de docente & 17 de $67(25,37 \%)$ & 16 de $32(50,00 \%)$ \\
\hline
\end{tabular}

Fonte: autores.

A maioria das respostas são oriundas de docentes, que representam $61,2 \%$ da amostra total, e a maior parte dos profissionais em geral são da Fatec Sorocaba: 45 respondentes ou $52,9 \%$ do total. A maior parte dos respondentes - 36 - possui dezesseis anos ou mais de serviços prestados à unidade Fatec, e outra faixa expressiva - 20 respondentes - possui entre nove e onze anos de experiência dentro da instituição. Isso evidencia a predominância de pessoas com carreiras consolidadas, o que também se cruza com a grande parte da amostra que estão entre as faixas etárias de 51 a 60 anos e a partir de 61 anos. Por outro lado, cerca de $16,5 \%$ dos respondentes estão na faixa entre 21 e 30 anos, e 12,9\% está na faixa entre 31 e 40 anos de idade, totalizando quase $30 \%$ de pessoas entre 21 e 40 anos, o que destaca uma presença evidente de jovens adultos no corpo de profissionais, especialmente no caso dos auxiliares de docente.

Agora, para facilitar a análise e leitura dos resultados, as atribuições elencadas anteriormente [seção Método] foram agrupadas em três principais grupos, quais sejam:

1- Atribuições técnico-operacionais específicas: nesse grupo, foram incluídas as atribuições $A, B, F, G, I, J, K, O$ e R;

2- Atribuições de relacionamento com os alunos: grupo no qual foram incluídas as atribuições C, D e E;

3- Outras atribuições: grupo contendo as atribuições $H, L, M, N, P, Q$ e S.

Dispondo-se desses grupos, apresenta-se a Figura 1, com os resultados obtidos para a percepção dos docentes quanto a tempo e importância das atribuições dos auxiliares de docente na Fatec.

Analisando a percepção geral dos docentes quanto ao grupo de atribuições técnicooperacionais específicas dos auxiliares de docente, evidencia-se que o grau de importância atribuído varia entre Alto e Muito Alto, denotando uma percepção predominantemente positiva, ao passo que o tempo necessário está geralmente entre os níveis Médio e Alto. Duas atribuições específicas, quais sejam ' $F$ ' - preparação do local de trabalho, dos materiais, ferramentas e etc. a serem utilizados, verificando as condições dos mesmos, o estado de conservação - e ' $\mathrm{K}$ ' - Providenciar e/ou confeccionar corpos de prova para ensaios de materiais de uso nos laboratórios e nas oficinas -, apresentaram percepção de alto consumo de tempo, destoando do nível médio geral, o que implica na visão geral de que os auxiliares de docente investem maior tempo nestas no que na maioria das outras pela percepção dos docentes. 
No caso das atribuições de relacionamento com os alunos, a figura 1d apresenta que duas estão com nível de importância Muito Alto pela maioria - relacionadas à realização dos procedimentos de maneira adequada -, e uma está com importância Alta relacionada à interpretação de dados em sala de aula para diferentes contextos. $O$ investimento de tempo está entre Médio a Alto, sendo mais próximo do nível Médio em geral.

Por fim, para o último grupo - outras atribuições -, os docentes atribuíram uma percepção de importância Alta, excetuando-se a atribuição ' $S$ ' - colaborar com o docente em programas de extensão universitária à comunidade -, que recebeu um grau de importância médio da maioria dos docentes. O grau de tempo investido pelo auxiliar de docente é apontado como médio para as atribuições desse grupo, sendo baixo apenas para a atribuição ' $H$ ' - comunicar ao superior hierárquico as irregularidades e os problemas constatados de qualquer ordem.

Figura 1 -

Resultados da amostra de docentes.
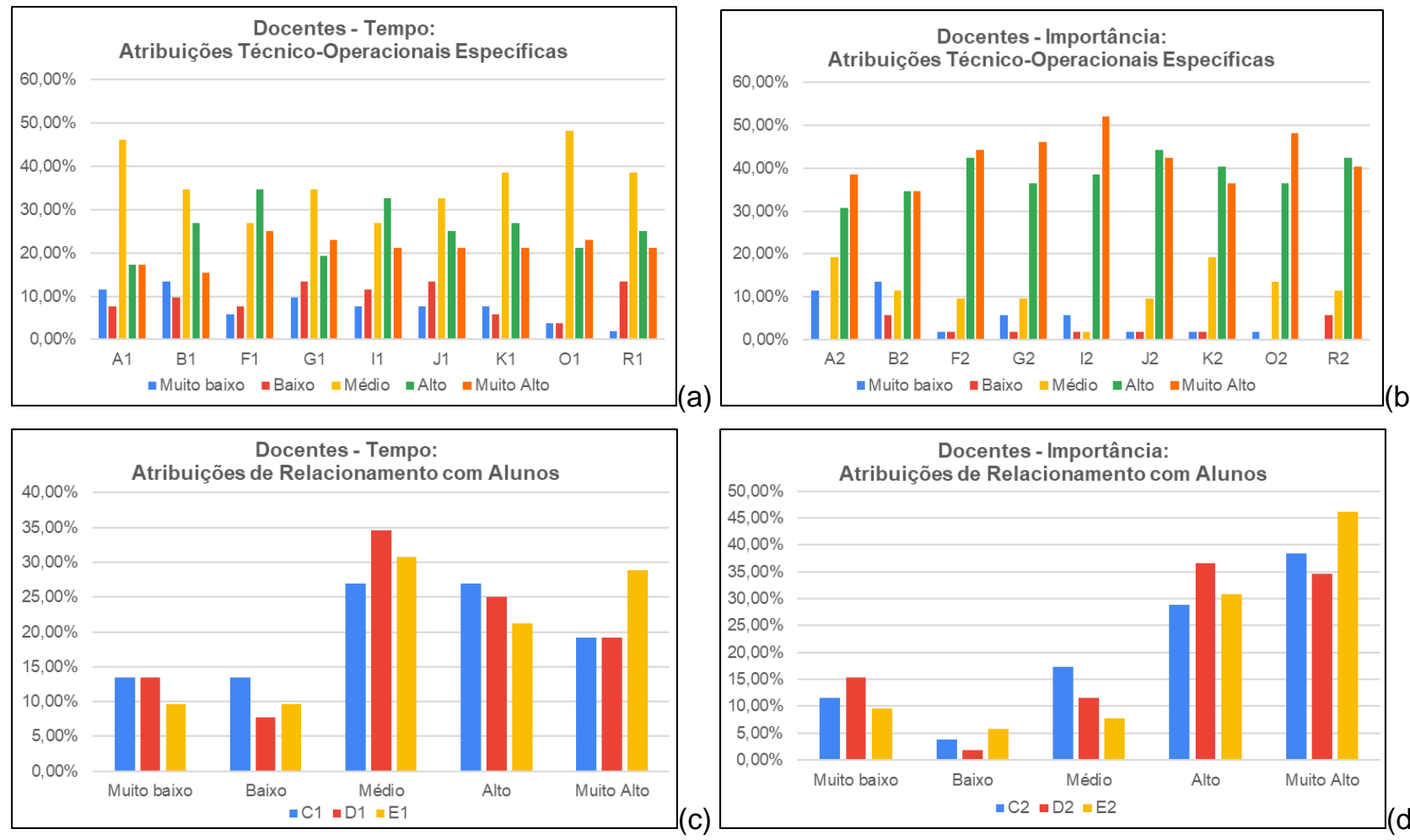

(b)
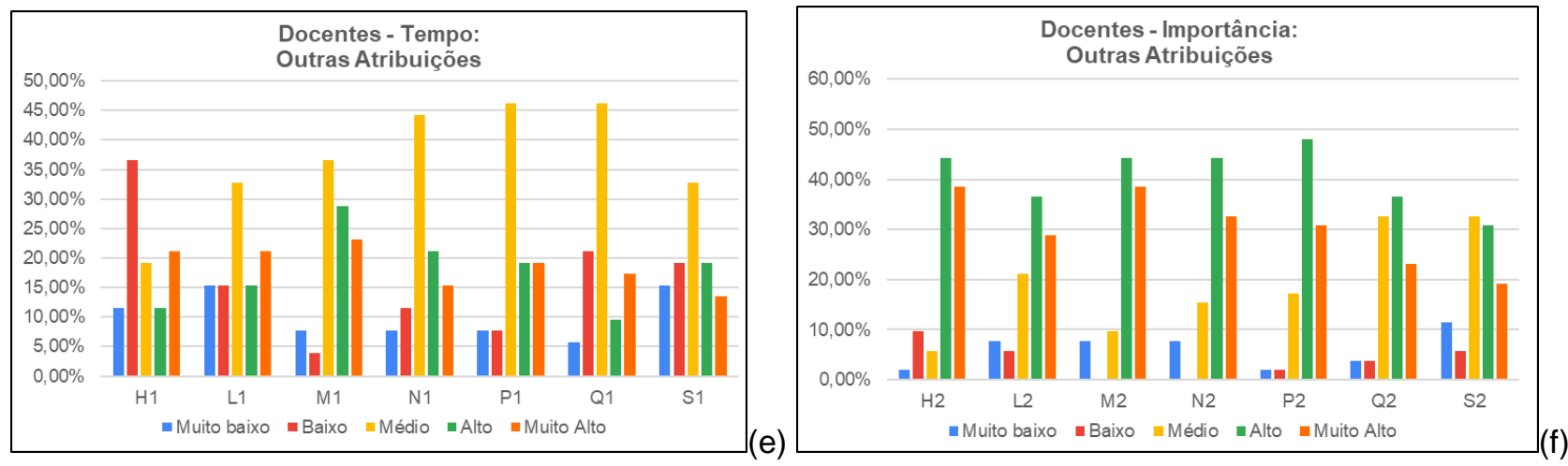

Fonte: autores. 
Percebe-se que, de maneira geral, a percepção por parte dos docentes é positiva no que se refere à atuação dos auxiliares de docente, com a maioria das atribuições entendidas como muito importantes e com demanda média de tempo investido. Especialmente as atribuições relacionadas às práticas técnicas e operacionais são consideradas relevantes e necessárias em sua atuação segundo a percepção dos docentes. Vale, entretanto, pontuar que não houve unanimidade em nenhuma situação, como por exemplo no caso da atribuição 'B' - efetuar demonstração das técnicas operacionais, manipulando ferramentas, máquinas, instrumentos e equipamentos -, com quase $15 \%$ dos respondentes apontando importância Baixa ou Muito Baixa, o que pode evidenciar que, na visão destes, ocorre alguma ineficiência na execução das funções, ou se trata de uma atribuição que não deveria ser considerada importante na atuação do auxiliar de docente, ou mesmo que essa atividade não é necessária para a sua disciplina ou laboratório.

Quanto à percepção dos auxiliares de docente, com as avaliações de tempo e importância para todos os grupos de atribuições, tem-se o que segue.

Figura 2 -

Resultados da amostra de auxiliares de docente.
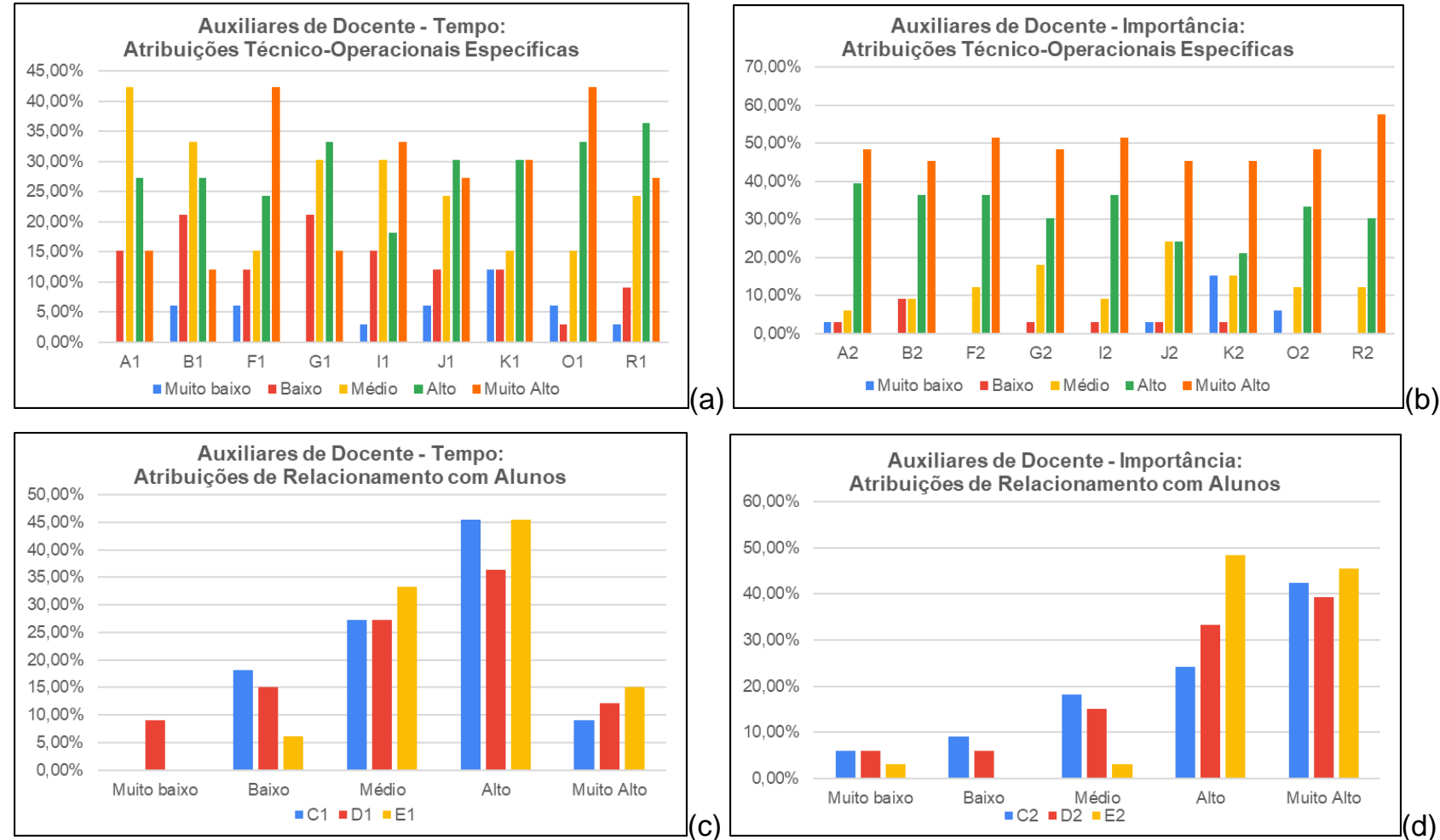

(c)
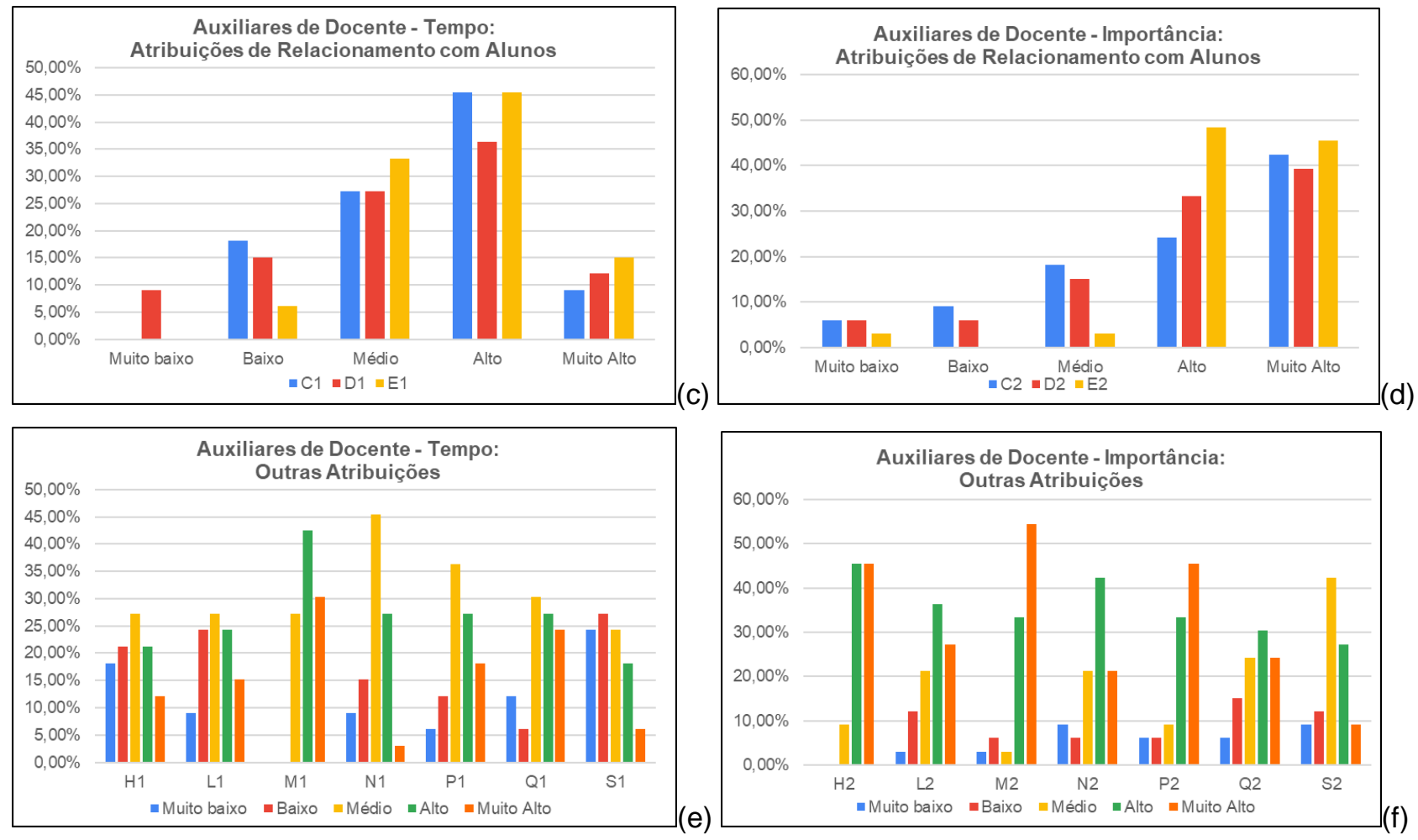

Fonte: autores. 
A maior parte dos respondentes apontou importância muito alta a todas as atribuições do grupo técnico-operacional específico, sendo pequenas as quantidades de respondentes que atribuíram importância baixa ou muito baixa. Quanto ao tempo, predomina-se a percepção de investimento alto a muito alto, o que difere dos docentes que predominantemente indicaram investimento médio de tempo -, e esse comportamento pode ser considerado adequado por ser esse grupo de atribuições aquele que mais demanda esforços e tempo dos auxiliares de docente frente à comunidade acadêmica.

Para o grupo de atribuições de relacionamento com os alunos, diferentemente da percepção dos docentes, a maioria dos respondentes atribuiu uma importância alta e não muito alta, dessas atribuições em sua rotina acadêmica. Ainda, a percepção de tempo investido nessas atividades por eles é majoritariamente alta, o que significa que são atribuições que demandam tempo, mas não são consideradas por eles como as mais importantes de sua atuação. Isso contrasta com Alhija e Fresko (2018), que ressaltaram que essas atribuições são as mais valorizadas pelos discentes e pela comunidade acadêmica das instituições de seu estudo, sendo assim as percebidas como mais importantes nesses grupos e às quais se esperava nível de importância muito alta pela maioria dos respondentes dos dois grupos profissionais.

Por fim, para o grupo de outras atribuições, observa-se a manutenção de uma percepção positiva em geral, estando a maior parte das atribuições categorizada com importância muito alta. A percepção sobre o investimento de tempo se mostrou muito variável, sem evidenciar claramente alguma tendência geral. A partir dos resultados tanto para docentes quanto para auxiliares de docente, percebe-se que as atribuições do grupo técnico-operacional específico foram consideradas de importância muito alta pelas maiorias, enquanto que as atribuições relacionadas a contatos com os alunos foram consideradas de importância alta a muito alta e as outras atribuições apresentaram alta variabilidade, mas sem fugir muito dessa faixa, o que denota uma percepção geral positiva da atuação dos auxiliares de docente nas autarquias.

A principal mudança entre a percepção desse grupo e dos docentes está na percepção do investimento de tempo nas atribuições: para a maioria dos auxiliares de docente, a maior parte das atividades demanda tempo alto a muito alto, o que difere da percepção dos docentes, que varia entre médio a alto. Da percepção dos auxiliares de docente, percebe-se que as atribuições relacionadas à manutenção das estruturas laboratórios, máquinas, equipamentos, reagentes e outros - são as que dispendem maior tempo em suas rotinas e são apontadas com alta ou muito alta importância tanto por docentes quanto por auxiliares de docente.

A quantidade de auxiliares de docente que apontou importância baixa ou muito baixa das atribuições em geral foi proporcionalmente menor do que a quantidade de docentes que fizeram tal apontamento. Porém, em alguns casos, como das atribuições ' $\mathrm{K}$ ' providenciar e/ou confeccionar corpos de prova para ensaios de materiais de uso nos laboratórios e nas oficinas -, 'L' - participar de reuniões sempre que convocado - e Q colaborar nos trabalhos gerais de instalação, manutenção e reparação, realizados na unidade de ensino -, entre os próprios auxiliares de docente chega-se a parcelas aproximadas de $18 \%, 15 \%$ e $21 \%$ respectivamente de percepções de importância baixa ou muito baixa [Fig. 2]. Algumas dessas atribuições, como a ' $\mathrm{K}$ ', podem ter passado por tal situação devido à não necessidade por algumas especialidades de auxiliares de

\begin{tabular}{|l|l|l|l|l|l|}
\hline Regae: Rev. Gest. Aval. Educ. & Santa Maria & v. 11 & n. 20 & e67834, p. 1-16 & 2022 \\
\hline
\end{tabular}


docente, ou seja, para aqueles profissionais que não desempenham tal atribuição por não ser inerente à sua área, a atribuição não se mostra relevante. Isso pode ocorrer também no caso das atribuições ' $L$ ' - reuniões - e 'Q' - trabalhos gerais, mas não é possível atestar isso de maneira categórica através do presente trabalho.

\section{Considerações finais}

O presente trabalho teve como objetivo apresentar a percepção a respeito da atuação dos auxiliares de docente no contexto da educação profissional e tecnológica praticada por duas instituições públicas de ensino superior brasileiras. O cargo de auxiliar de docente apresentou certa variabilidade de funções ao longo do tempo e, dessa forma, é válido realizar um estudo sobre as percepções dos envolvidos e dos correlacionados com esses profissionais.

Evidencia-se que a atuação dos auxiliares de docente é percebida de maneira positiva pelos respondentes, o que ocorre especialmente com as atribuições técnicooperacionais específicas e de relacionamento com os alunos, que apresentaram níveis elevados de importância percebidos pela maioria dos respondentes e reforçam assim 0 papel principal esperado do profissional na estrutura do ensino tecnológico. O grupo de auxiliares de docente enxergou a atuação dos mesmos de maneira ainda mais positiva que os docentes, que, por sua vez, tiveram quantidades entre $30 \%$ e $50 \%$ de respondentes que indicaram percepção de importância Muito Alta na maior parte das atribuições.

O grupo de outras atribuições, relacionadas a atividades gerais e de menor demanda, é enxergado como secundário e sua importância ficou entre média e alta pela maioria dos respondentes docentes, ou especialmente em alta para a percepção dos auxiliares de docente. Também foi possível, a partir da presente pesquisa, compreender melhor a atuação do auxiliar de docente, sendo válido pontuar, considerando o contexto histórico, que a atuação ora avaliada se aproxima muito à do antigo Instrutor e está mais distante das atribuições do antigo Auxiliar de docente, mais voltado a funções administrativas [especialmente presente no grupo de outras atribuições].

O cargo se mostra relevante na estrutura e se propõe a consolidar a educação tecnológica de maneira mais assertiva, uma vez que se trata de um interlocutor com diversos agentes e um eficiente facilitador do ensino prático e teórico. Porém, ainda se observa uma variabilidade de atribuições entre os contextos mais técnicos e práticos ou administrativos e de simples suporte, o que decorre de seu contexto histórico e implica em percepções diversas entre os grupos em questão.

\section{Referências}

ALEXANDER, Zachary Wahl; SMITH, Matthew D. Curtner. Influence of negotiations on graduate teaching assistants' instruction within university activity courses. Journal of Teaching in Physical Education, Champaign, IL, v. 37, 2018, p. 164-174.

ALHIJA, Fadia Nasser-Abu; FRESKO, Barbara. Graduate teaching assistants: how well do their students think they do? Assessment and Evaluation on Higher Education, Tel Aviv, Israel, v. 1, 2018, p. 1-12. 
AYENI, Abiodun Olumide. World wide comparism of technical and vocational education: lessons for nigerian technical and vocational education sector. Journal of Education and Practice, Ibadan, Nigeria, v. 6, n. 30, 2015, p. 103-110.

BARAKI, Atakilt Hagos; KEMENADE, Everard van. Effectiveness of technical and vocational education and training (TVET): insights from Ethiopia's reform. The TQM Journal, v. 25, n. 5, 2013, p. 492-506.

BRASIL. Constituição Federal da República Federativa do Brasil. Brasília: Senado Federal, 1988.

BRASIL. Lei n. 9.394, de 20 de dezembro de 1996: estabelece as diretrizes e bases da educação nacional. Brasília: Senado Federal, 1996

BRASIL. Lei n. 11.741, de 16 de julho de 2008. Disponível em: http://www.planalto.gov.br/ccivil_03/_ato2007-2010/2008/lei//11741.htm. Acesso em 2 set. 2021.

BRASIL. Lei n. 13.415, de 16 de fevereiro de 2017. Disponível http://www.planalto.gov.br/ccivil_03/_ato2015-2018/2017/lei//13415.htm. Acesso em 2 set. 2021.

CEETEPS. Deliberação Ceeteps n. 3, de 18-7-2013: aprova o Regimento Comum das Escolas Técnicas Estaduais do Centro Estadual de Educação Tecnológica Paula Souza. 2013. Disponível em: http://www.portal.cps.sp.gov.br/etec/regimento-comum/regimentocomum-2013.pdf. Acesso em: 5 set. 2019.

CEETEPS. Deliberação Ceeteps n. 8, de 10-7-2014: regulamenta as atribuições dos empregos públicos, abrangidos pelo Plano de Carreira, de Empregos Públicos e Sistema Retribuitório, de que trata o artigo 40 da Lei Complementar n. 1.044, de 13 de maio de 2008, alterada pela Lei Complementar n. 1.240, de 22 de abril de 2014 e dá providências correlatas. São Paulo: Ceeteps, 2014.

CHOU, Chih-Yueh; HUANG, Bau-Hung; LIN, Chi-Jen. Complementary machine intelligence and human intelligence in virtual teaching assistant for tutoring program tracing. Computers \& Education, Taipei, Taiwan, v. 57, 2011, p. 2303-2312.

FEITOZA, Andreza Santos; DUDUCHI, Marcelo. Observando a Educação Profissional e Tecnológica: momentos, contextos e visões. WORKSHOP DE PÓS-GRADUAÇÃO E PESQUISA DO CENTRO PAULA SOUZA, 11, 2016. Anais ... CPS, 2016.

HERNÁNDEZ, Vadim Aguilar. Desafíos de la investigación en la enseñanza técnica profesional en Cuba. Revista MENDIVE, Pinar del Río, Cuba, v. 14, n. 1, 2016, p. 1-86.

INEP. Censo da educação superior 2019. Disponível em: https://download.inep.gov.br/educacao_superior/censo_superior/documentos/2020/Aprese ntacao_Censo_da_Educacao_Superior_2019.pdf. Acesso em: 2 set. 2021.

JUSTICE, Nicola; ZIEFFLER, Andrew; GARFIELD, Joan. Statistics graduate teaching assistants' beliefs, practices and preparation for teaching introductory statistics. Statistics Education Research Journal, Minnesota, EUA, v. 16, n. 1, 2017, p. 294-319.

LIAO, Min-Yu. Taiwanese student's perceptions of teaching assistants' effectiveness ingroup work education. Social Work Education, Taipei, Taiwan, v. 37, n. 2, p. 2018, 250264.

MAGALHÃES, Guilherme Lins de; CASTIONI, Remi. Educação Profissional no Brasil expansão para quem? Ensaio: Avaliação de Políticas Públicas em Educação, Brasília, v. 1, n. 1, 2019, p. 1-23. 
MALARA, Maria Bernadete da Silva. Os saberes docentes do professor universitário do curso introdutório de Estatística expressos no discurso dos formadores. São Paulo: Unesp, 2008. 309f. Tese (Doutorado) - Instituto de Geociências e Ciências Exatas, Universidade Estadual Paulista.

MARSHMAN, Emily; SAYER, Ryan; HENDERSON, Charles; YERUSHALMI, Edith; SINGH, Chandralekha. The challenges of changing teaching assistants' grading practices: requiring students to show evidence of understanding. Can. J. Phys., Pittsburgh, EUA, v. 96, 2018, p. 420-437.

MAURER, Markus. Structural elaboration of technical and vocational education and training systems in developing countries: the cases of Sri Lanka and Bangladesh. Comparative Education, Zurique, Suíça, v. 48, n. 4, 2012, p. 487-503.

MEC. Sítio oficial. 2020. Disponível em: portal.mec.gov.br. Acesso em: 2 set. 2021.

NOORUDDIN, Shirin. Technical and Vocational Education and Training for Economic Growth in Pakistan. Journal of Education and Educational Development, Karachi, Paquistão, v. 4, n. 1, 2017, p. 130-141.

OKUMU, Ibrahim Mike; BBAALE, Edward. Technical and vocational education and training in Uganda: a critical analysis. Development Policy Review, Kampala, Uganda, v. 00, n. 00, 2018, p. 1-15.

OLIVEIRA, Antonio Cardoso; CÓsSIO, Maria de Fátima. O atual cenário da Educação Profissional no Brasil. CONGRESSO NACIONAL DE EDUCAÇÃO, 11, 2013. Anais ... Curitiba: PUCPR, 2013.

PETEROSSI, Helena Gemignani. A gestão dos cursos de tecnologia: rupturas e continuidades. CONGRESSO MUNDIAL DE TRANSDISCIPLINARIDADE, 2, 2005. Anais ... São Paulo: PUCSP, 2005.

QUEVEDO, Margarete de. Educação Profissional no Brasil: formação de cidadãos ou de mão de obra para o mercado de trabalho? Revista de Humanidades, Tecnologia e Cultura da Fatec Bauru, Bauru, v. 1, n. 1, 2011, p. 147-160.

REEVES, Todd D; AD, Gili Marbach; MILLER, Kristen R; RIDGWAY, Judith; GARDNER, Grant E; SCHUSSLER, Elisabeth E; WISCHUSEN, E. William. A conceptual framework for graduate teaching assistant professional development evaluation and research. Life Sciences Education, DeKalb, Illinois, v. 15, 2016, n. 1-9.

RUTLEDGE, Chayla D; BULLARD, Morgan B; EVANS, Patricia Kohler; FILER, Janet D. The effects of cue-do-review on teaching assistant and student perceptions of learning. Education, Alabama, EUA, v. 39, n. 4, 2018, p. 187-196.

SÃO PAULO. Decreto n. 17.027, de 19 de maio de 1981: aprova o Regimento do Centro Estadual de Educação Tecnológica Paula Souza. Disponível em: https://www.al.sp.gov.br/norma/55842. Acesso em: 2 set. 2021.

SÃO PAULO. Lei complementar n. 1.044, de 13 de maio de 2008: institui o plano de carreiras, de empregos públicos e sistema retribuitório dos servidores do Centro Estadual de Educação Tecnológica Paula Souza. Disponível em: https://www.al.sp.gov.br/repositorio/legislacao/lei.complementar/2008/lei.complementar1044-13.05.2008.html. Acesso: em 2 set. 2021. 
SÃO PAULO. Lei complementar n. 1.240, de 22 de abril de 2014: altera a lei complementar n. 1.044, de 2008, que institui o plano de carreiras, de empregos públicos e sistema retribuitório dos servidores do Ceeteps e dá outras providências. Disponível em: https://www.al.sp.gov.br/repositorio/legislacao/lei.complementar/2014/lei.complementar1240-22.04.2014.html. Acesso em: 2 set. 2021.

SÃO PAULO. Lei complementar n. 1.343, de 26 de agosto de 2019: altera a Lei Complementar n. 1.044, de 2008, que institui o plano de carreiras, de empregos públicos e sistema retribuitório dos servidores do Ceeteps e dá outras providências. Disponível em: https://www.al.sp.gov.br/norma/191324. Acesso em: 2 set. 2021.

UEMURA, Marise Regina Barbosa. Fatores determinantes no desempenho das escolas de ensino profissionalizante integrado ao médio: um estudo de caso dos municípios de Cotia e São Roque. São Paulo: USP, 2016. 175f. Dissertação (Mestrado em Administração) - Faculdade de Economia, Administração e Contabilidade, Universidade de São Paulo.

VIEIRA, Alboni Marisa Dudeque Pianovski; SOUZA JÚNIOR, Antônio de. A Educação profissional no Brasil. Revista Interacções, Curitiba, v. 1, n. 40, 2016, p. 152-169.

WANG, Anyi; GUO, Dong. Technical and vocational education in China: enrolment and socioeconomic status. Journal of Vocational and Educational Training, Nova lorque, EUA, v. 1, n. 1, 2018, p. 1-18.

WREN, Alison. Understanding the role of the teaching assistant: comparing the views of pupils with SEN and TAs with mainstream primary schools. Support for Learning, Londres, Reino Unido, v. 32, n. 1, 2017, p. 4-19.

Julio Francisco Rodrigues de Sousa é professor na Faculdade de Tecnologia de São Paulo.

Orcid: https://orcid.org/0000-0002-5222-719X.

Endereço: Rua dos Bandeirantes, 169 - 01124-010 - São Paulo - SP - Brasil.

E-mail: link julio@hotmail.com.

Marília Macorin de Azevedo é professora no Centro Estadual de Educação Tecnológica Paula Souza.

Orcid: https://orcid.org/0000-0003-0225-8155.

Endereço: Rua dos Bandeirantes, 169 - 01124-010 - São Paulo - SP - Brasil.

E-mail: marilia.azevedo@fatec.sp.gov.br.

Paulo Roberto Prado Constantino é professor no Centro Estadual de Educação Tecnológica Paula Souza.

Orcid: https://orcid.org/0000-0002-4612-4063.

Endereço: Rua dos Bandeirantes, 169 - 01124-010 - São Paulo - SP - Brasil.

E-mail: paulo.constantino@cps.sp.gov.br. 
Critérios de autoria: os autores conceberam a ideia. Julio Francisco Rodrigues de Sousa coletou os dados, analisou e preparou a primeira versão. Todos os autores discutiram os resultados e contribuíram para a versão final do manuscrito.

Recebido em 27 de setembro de 2021.

Aceito em 3 de janeiro de 2022.

(c) (i) $(5)$ 